\title{
Neutropenia and Infection Prophylaxis in Childhood Cancer
}

\author{
Stephanie Villeneuve ${ }^{1} \cdot$ Catherine Aftandilian $^{2}$
}

Accepted: 27 September 2021 / Published online: 1 March 2022

(c) The Author(s), under exclusive licence to Springer Science+Business Media, LLC, part of Springer Nature 2022

\begin{abstract}
Purpose of Review Pediatric oncology patients frequently experience episodes of prolonged neutropenia which puts them at high risk for infection with significant morbidity and mortality. Here, we review the data on infection prophylaxis with a focus on both pharmacologic and ancillary interventions. This review does not include patients receiving hematopoietic stem cell transplantation.

Recent Findings Patients with hematologic malignancies are at highest risk for infection. Bacterial and fungal prophylaxis decrease the risk of infection in certain high-risk groups. Ancillary measures such as ethanol locks, chlorhexidine gluconate baths, GCSF, IVIG, and mandatory hospitalization do not have enough data to support routine use. There is limited data on risk of infection and role of prophylaxis in patients receiving immunotherapy and patients with solid tumors. Patients with Down syndrome and adolescent and young adult patients may benefit from additional supportive care measures and protocol modifications.

Summary Consider utilizing bacterial and fungal prophylaxis in patients with acute myeloid leukemia or relapsed acute lymphoblastic leukemia. More research is needed to evaluate other supportive care measures and the role of prophylaxis in patients receiving immunotherapy.
\end{abstract}

Keywords Pediatric oncology $\cdot$ Infection prophylaxis $\cdot$ Neutropenia $\cdot$ Antibiotic prophylaxis $\cdot$ Antifungal prophylaxis . Ethanol locks $\cdot$ Chlorhexidine gluconate baths $\cdot$ IVIG $\cdot$ GCSF

\section{Introduction}

Survival in pediatric oncology has improved significantly over the past 50 years due to improvements in chemotherapy and supportive care $[1,2]$. Current therapies are often intensive and can result in prolonged periods of neutropenia which put patients at high risk for infection with significant morbidity and mortality [3]. A review of infection-related mortality (IRM) in patients with newly diagnosed acute lymphoblastic leukemia (ALL) found that IRM accounted for $30 \%$ of deaths and $64 \%$ of treatment-related mortality.

This article is part of the Topical Collection on Pediatric Oncology

Catherine Aftandilian

aftandil@stanford.edu

1 Paediatric Haemotology/Oncology, Dalhousie University and the IWK Health Centre, 5850/5980 University Avenue, Halifax, NS B3K 6R8, Canada

2 Pediatric Hematology/Oncology, Stanford University, 1000 Welch Rd, Palo Alto, CA 94304, USA
The majority of deaths were due to bacterial infections (68\%), but a substantial proportion were also secondary to fungal and viral infections (20\% and $12 \%$, respectively). This review article will focus on common strategies for infection prophylaxis including bacterial and fungal prophylaxis, as well ancillary measures such as ethanol locks, chlorhexidine gluconate baths (CHB), granulocyte colony stimulating factor (GCSF), intravenous immunoglobulin (IVIG) supplementation, and mandatory hospitalization in certain high-risk groups.

When evaluating the utility of infection prophylaxis, it is important to consider both anticipated and unanticipated consequences. While prevention of infections is paramount, concerns exist around promoting resistant organisms [4], as well as increased secondary infections such as $C$. difficile infection (CDI) [5], potential alterations to the microbiome with associated dysbiosis [6], and drug toxicity which may impact chemotherapy dosing [7]. Thus, while the intention of prophylaxis is to reduce harm, the risks and benefits need to be evaluated carefully in each individual patient based on their underlying disease, phase of therapy, other risk factors 
for infection, patient/family preference, and potential benefits/toxicities of prophylaxis options.

\section{Bacterial Prophylaxis}

There are multiple risk factors associated with the development of bacterial infections in pediatric oncology patients including recent placement of a central venous catheters (CVCs) [8], compromise of mucosal barriers (e.g. mucositis, typhlitis) [9], and myelosuppression [8, 9]. Underlying diagnosis is also important as children with hematologic malignancies have an increased risk relative to children with solid tumors $[9,10]$. Strategies to mitigate the rate of infection have been reported in both adult and pediatric oncology populations as bacterial infection remains a significant cause of morbidity and mortality. Bacterial prophylaxis in adults is an effective means to decrease risk of fever, documented infections, and mortality in patients with chemotherapyinduced myelosuppression [11].

Within pediatrics, both observational and randomizedcontrolled trials demonstrate that antibiotic prophylaxis is associated with decreased risk of fever, decreased exposure to broad spectrum antibiotics, decreased CDI, and decreased bacteremia in certain high-risk groups (Table 1). Three studies have evaluated antibiotic prophylaxis in patients with newly diagnosed ALL. In a prospective cohort of 344 patients, Wolf et al. found that levofloxacin prophylaxis was associated with decreased febrile neutropenia, decreased bacteremia, decreased CDI, and decreased exposure to broad-spectrum antibiotics [12]. In addition, there are two recent randomized-controlled trials reviewing the role for antibiotic prophylaxis in patients with ALL. Widjajanto et al. conducted a double-blind, randomized trial of ciprofloxacin vs. placebo in 110 children with ALL undergoing induction chemotherapy in a low resource setting [13]. This study was unique due to the high rate of toxic death (23\%) and therapy refusal/abandonment (35\%) during induction therapy reported among patients in Indonesia. Contrary to other reported studies, Widjajanto et al. found a trend toward higher rates of fever $(p=0.07)$, and toxic death $(p=0.05)$ in the children receiving ciprofloxacin. There were 11 deaths in the treatment group vs. 3 deaths among the placebo group. Of the 11 deaths reported among the treatment group, 2 were attributed to sepsis vs. 3 within the placebo group. Other causes of death reported included varicella, intracranial hemorrhage, multi organ failure, shock, and transfusion reaction. Lastly, there were 2 patients who had no available data. Due to the unique patient population, these results may not be applicable in high-resource settings. Laoprasopwattana et al. randomized 95 children with ALL undergoing induction to ciprofloxacin vs. placebo and found lower rates of fever (50\% vs. $73 \%)$ in those with neutropenia who were receiving ciprofloxacin prophylaxis. Importantly, they also found an increased rate of resistance to ciprofloxacin in those receiving prophylaxis [14].

Alexander et al. published a multi-center, open-label, randomized controlled trial evaluating levofloxacin prophylaxis in 195 patients with acute myeloid leukemia (AML) and relapsed ALL. In this study, short-term levofloxacin prophylaxis resulted in a significant decrease in bacterial infection $(21.9 \%$ vs. $43.4 \% \mathrm{p}=0.001)$ without increasing adverse events such as CDI, fungal infection, or rates of resistant organisms [15•]. Of note, this study followed the use of antibiotics through two chemotherapy cycles, and therefore, it is difficult to extrapolate the potential implications of levofloxacin prophylaxis over a longer duration. The results of this trial are supported by a systematic review by Egan et al., which included 113 randomized or quasi-randomized studies comparing any systemic antibiotic prophylaxis with placebo in adult and pediatric patients receiving chemotherapy or undergoing HSCT for any indication. While the studies included were highly heterogenous, the review found that fluoroquinolone prophylaxis reduced the incidence of bacteremia without increasing the risk of CDI or fungal infections; however, one important point of differentiation was that increased rates of resistant organisms were seen across populations receiving prophylaxis. This review also documented the effectiveness of cephalosporins and trimethoprim-sulfamethoxazole in reducing bacteremia. Importantly, the use of antibiotic prophylaxis did not have a significant impact on overall mortality [16, 17••].

The role for antibiotic prophylaxis has been carefully weighed by multiple groups to form overarching clinical practice guidelines (Table 2). Among these are the recently published recommendations from the 8th European Council on Infections in Leukemia which weighed data from the most recent clinical trials as well as observational studies. The group recommended against routine bacterial prophylaxis in patients with hematologic malignancies or undergoing hematopoietic stem cell transplant (HSCT), citing that clinical trial data is not sufficient to support a recommendation for prophylaxis and that antimicrobial resistance must be considered [18]. In contrast, the Children's Oncology Group (COG) recommended consideration of systemic bacterial prophylaxis in patients with AML or relapsed ALL who are anticipated to be neutropenic for 7 days or longer. They recommend against prophylactic antibiotics in patients with a new diagnosis of ALL. The rationale for recommending against prophylaxis in the ALL population is due to a paucity of clinical trial evidence denoting benefit in a high resource population $[17 \bullet \bullet]$. 


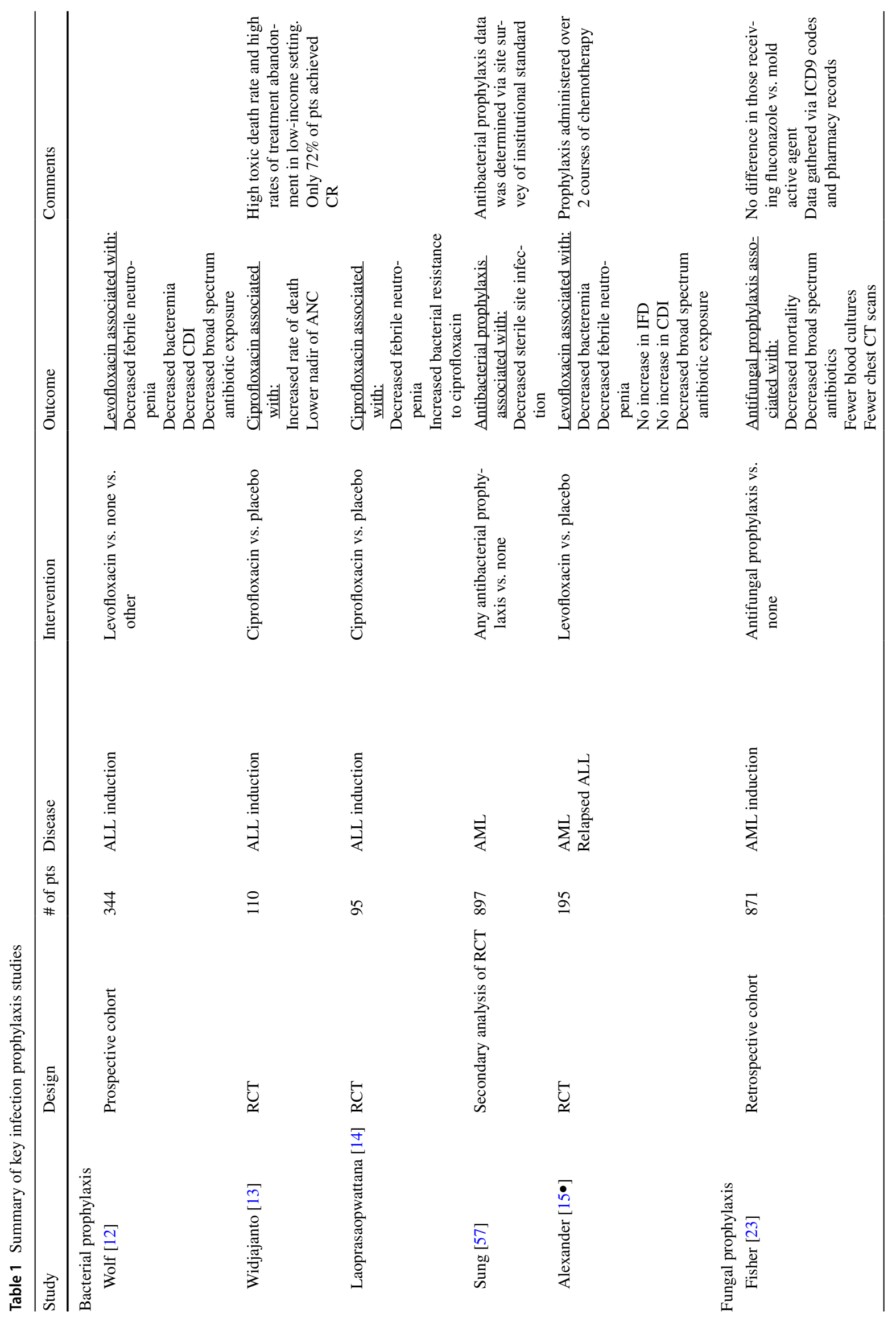




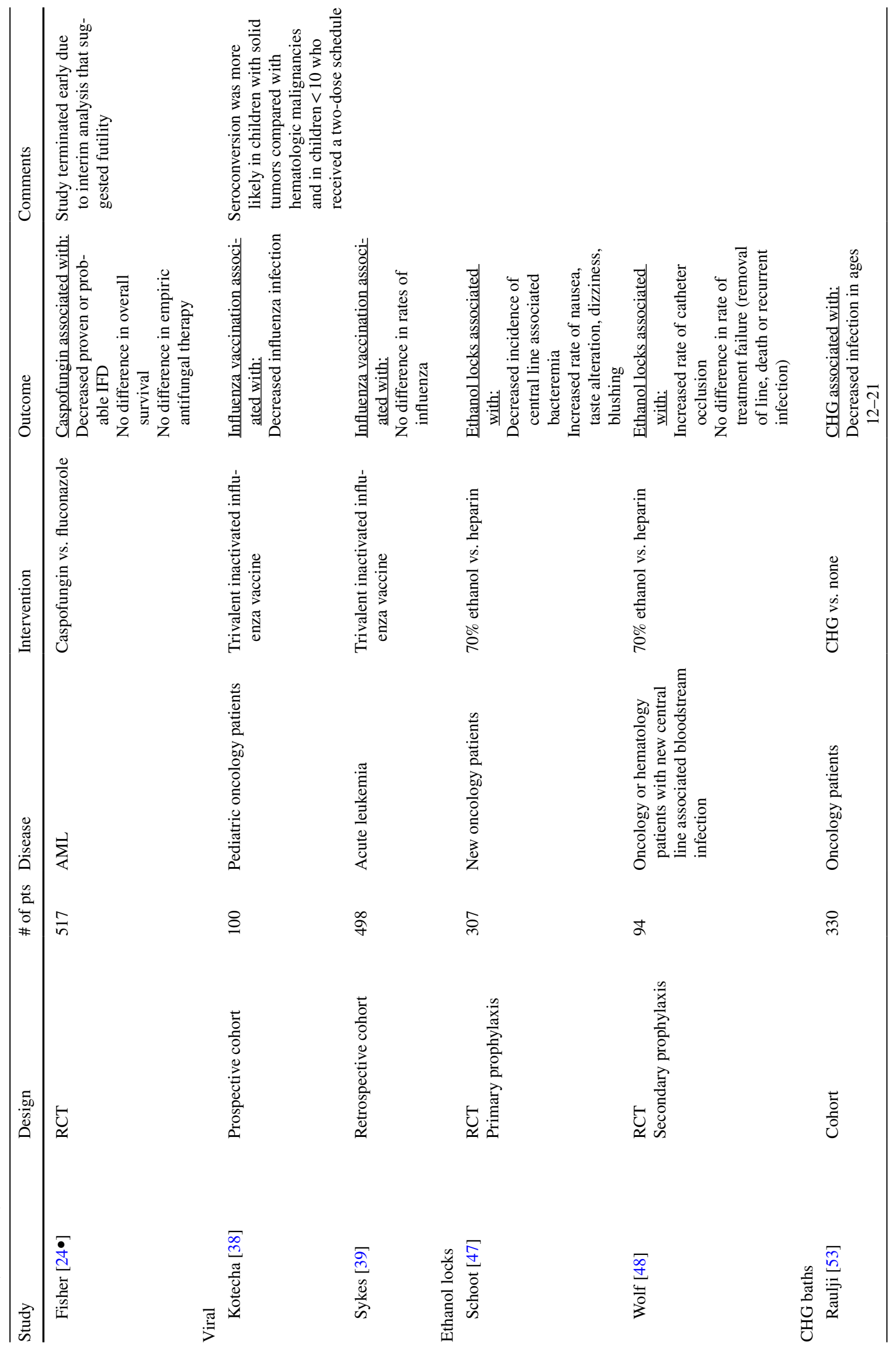




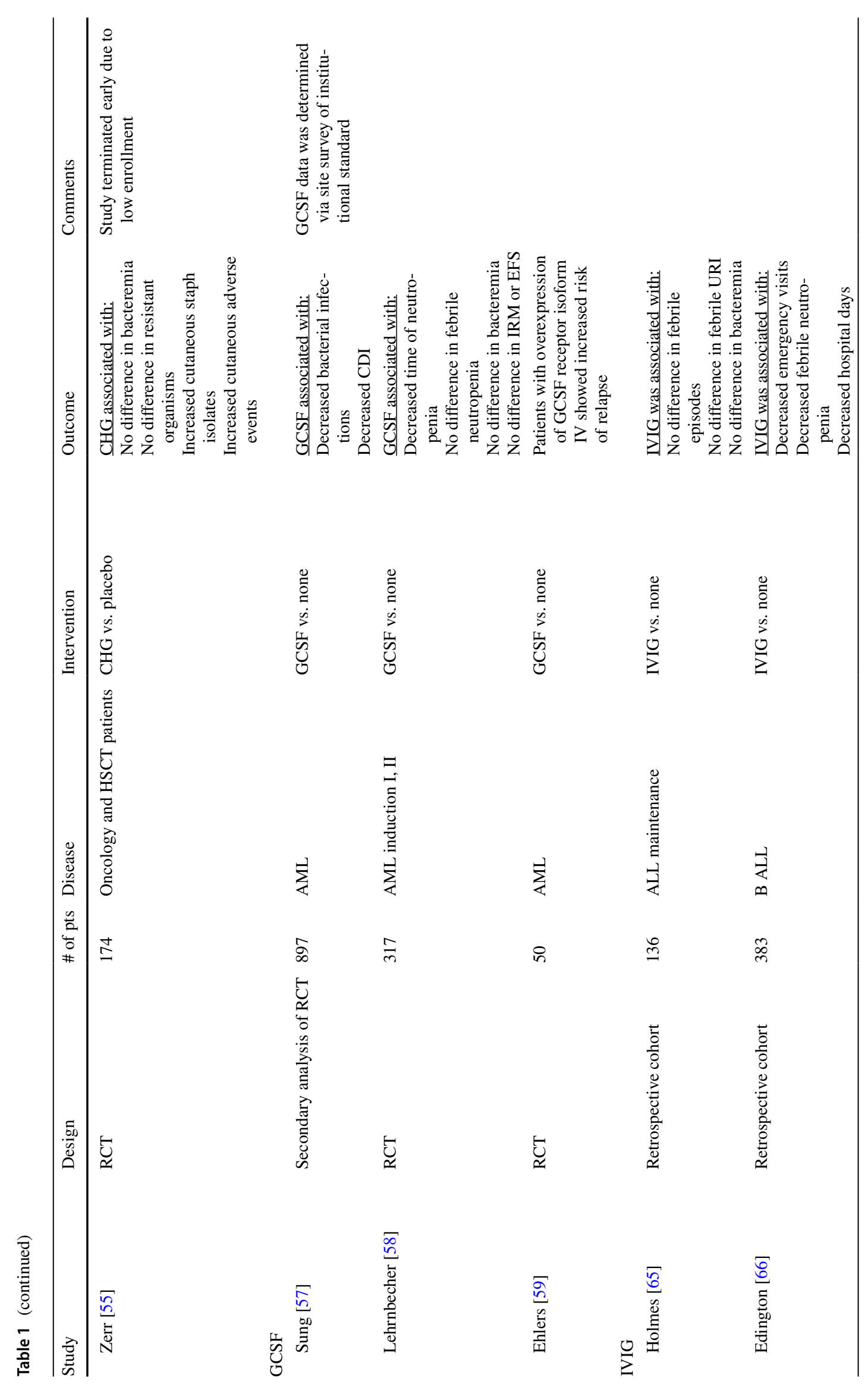


676

Current Oncology Reports (2022) 24:671-686

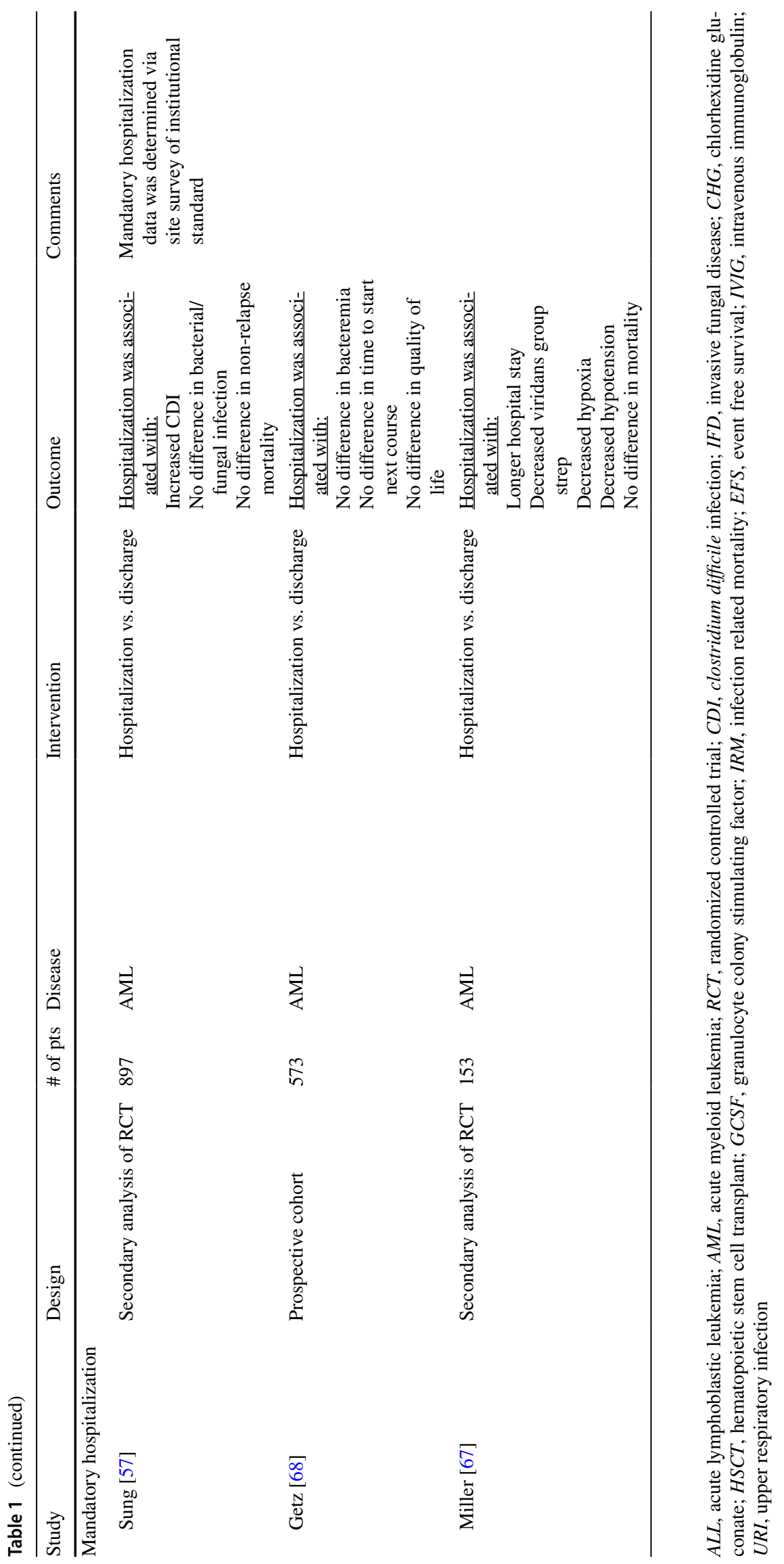

Springer 


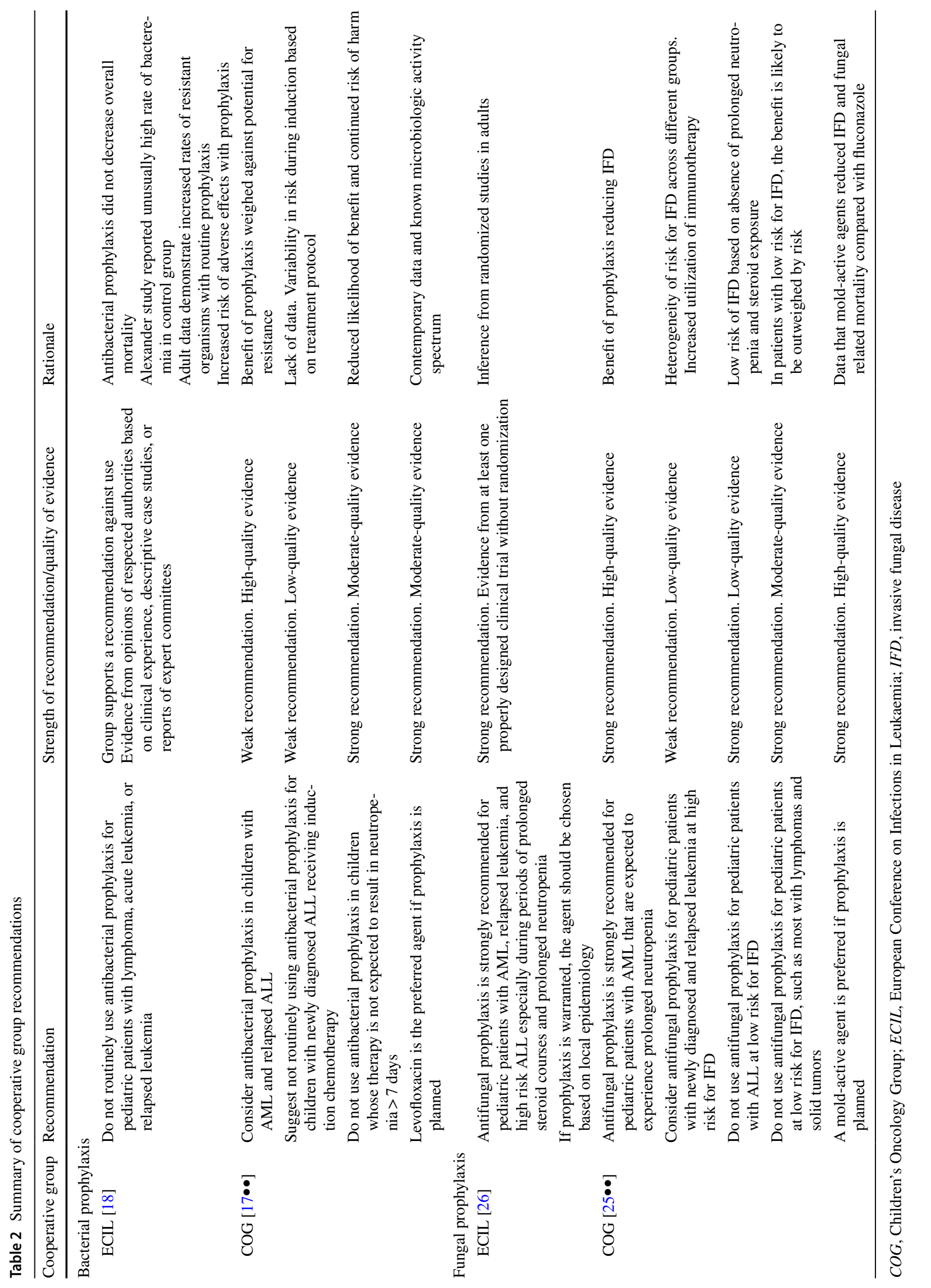




\section{Fungal Prophylaxis}

Invasive fungal disease (IFD) causes significant morbidity and mortality in pediatric oncology, especially in those with acute leukemia $[19,20]$. In patients with newly diagnosed ALL, IFD was responsible for $20 \%$ of infectionrelated deaths [3]. IFD can be difficult to diagnose and treat $[21,22]$, so prophylaxis is an especially important strategy to decrease morbidity and mortality. Fisher et al. conducted a systematic review of risk factors for IFD in pediatric oncology. Duration and depth of neutropenia as well as exposure to high-dose steroids, underlying diagnosis of AML or ALL, and increasing age were all risk factors for IFD [20]. Increasing age had not been previously reported. It is unclear if age itself is a risk factor or if age is associated with other factors such as increasing intensity of chemotherapy or altered pharmacokinetics which predispose to IFD.

Patients with AML are at particularly high risk for IFD as their chemotherapy results in prolonged periods of severe neutropenia [20]. As a result of their high risk, several studies have evaluated the utility of antifungal prophylaxis in pediatric AML (Table 1). In a cohort of 871 pediatric patients with AML from the Pediatric Health Information System database, Fisher et al. found that antifungal prophylaxis was associated with a reduced rate of induction mortality [23]. Interestingly, antifungal prophylaxis was also associated with decreased exposure to broad spectrum antibiotics as well as decreased resource utilization such as fewer blood cultures and chest CTs. There was no difference in mortality, antibiotic exposure or resource utilization in those receiving fluconazole vs. those receiving prophylaxis with a mold-active agent, defined as voriconazole, posaconazole, itraconazole, echinocandins, or amphotericin B products.

There is only one randomized, controlled trial of antifungal prophylaxis in the pediatric oncology population. Fisher et al. randomized 517 patients with AML to caspofungin or fluconazole prophylaxis during periods of neutropenia [24•]. Caspofungin prophylaxis was associated with a decreased risk of proven or probable IFD compared with fluconazole prophylaxis ( $3.1 \%$ vs. $7.2 \%, p=0.03)$. There was no difference in overall survival or in utilization of empiric antifungal therapy. This study was terminated early due to an interim analysis that suggested futility.

There are currently two clinical practice guidelines specific to antifungal prophylaxis in the pediatric oncology

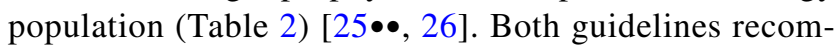
mend antifungal prophylaxis for children and adolescents receiving treatment for AML. Lehrnbecher et al. recommend antifungal prophylaxis with an echinocandin or a mold-active azole [25••]. Groll et al. provide dosing recommendations for various antifungals but do not specify a particular antifungal [26]. Recommendations for antifungal prophylaxis differ for pediatric patients with ALL. Lehrnbecher et al. recommend considering prophylaxis in certain children with ALL who may be at increased risk of IFD including those with relapsed disease. This weak recommendation is based on lack of baseline data on IFD incidence in children with both newly diagnosed and relapsed ALL as well as lack of any randomized controlled trials in this patient population. In contrast, Groll et al. strongly recommend antifungal prophylaxis for patients with relapsed ALL and those with high risk ALL based on randomized studies from the adult population.

\section{Pneumocystis jirovecii pneumonia}

Pneumocystis jirovecii pneumonia (PJP) is an opportunistic fungal infection that causes rapidly progressive pneumonia in immunocompromised patients. Prophylaxis against PJP has been standard of care in pediatric oncology patients after a landmark 1977 randomized, double-blind, placebocontrolled study found that trimethoprim sulfamethoxazole (TMP-SMX) was highly effective [27]. For patients who do not tolerate TMP-SMX, pentamidine, dapsone, and atovaquone are alternative options, but are associated with increased risk of PJP compared with TMP-SMX [28].

While the risk for PJP pneumonia is highest in patients with leukemia and lymphoma [29], prophylaxis is also recommended for children with solid tumors undergoing chemotherapy that is likely to cause lymphopenia [30]. Data in patients with HIV shows that the risk of PJP is highest in those with CD4 counts under 200, and one study from the solid organ transplantation population found that odds of PJP infection were significantly increased when absolute lymphocyte count (ALC) was $<500$ [31]. There is no data in pediatric oncology patients suggesting a specific threshold of CD4 count or ALC. As a result of this lack of data, optimal duration of PJP prophylaxis is unknown. One guideline suggests continuing until 3 months after the end of therapy and normalization of lymphocyte count [30].

\section{Viral Prophylaxis}

There is a paucity of data on viral prophylaxis in the pediatric oncology population. This partly due to a lack of information on the frequency, severity, and impact of viral infections in this population. Dixon et al. conducted a retrospective review of viral surveillance in pediatric patients with newly diagnosed or relapsed ALL or AML [32]. Of the 111 patients included, $36 \%$ had at least one blood PCR positive for EBV, CMV or adenovirus. Interestingly, the majority of patients with a positive viral PCR did not receive any targeted antiviral therapy. 
Patients were more likely to receive treatment for their viral infection if they had relapsed disease, infantile or high-risk ALL. Patients who received IVIG prophylaxis were less likely to experience a positive viral PCR $(16.9 \%$ vs. $63 \%$ p < 0.01$)$.

Buus-Gehrig et al. conducted a systematic review of viral infections in pediatric acute leukemia [33]. While there are multiple reports of severe viral infection causing either death or permanent disability, these cases are rare, and there is not yet enough data to warrant empiric prophylaxis in pediatric oncology patients.

\section{Influenza Vaccination}

In contrast to antiviral prophylaxis, there is widespread adoption of influenza vaccination across pediatric oncology centers [34]. Multiple studies have found that pediatric oncology patients experience immune response to vaccination, but these responses are weaker than in healthy children [35-38]. Kotecha et al. found that $2 \%$ of vaccinated pediatric oncology patients developed influenza infection compared with $6.8 \%$ of unvaccinated controls for an estimated vaccine effectiveness of $72 \%$ (95\% confidence interval 26-94\%) [38]. In contrast, Sykes et al. found that influenza vaccination did not decrease the risk of influenza infection in a cohort of children with ALL (Table 1) [39]. Despite potential lack of efficacy in certain populations, the American Academy of Pediatrics and Center for Disease Control still recommend yearly influenza vacations in pediatric oncology patients based on potential benefit with low risk of reaction.

\section{SARS CoV-2 Vaccination}

Severe acute respiratory syndrome coronavirus-2 (SARSCoV-2) caused a global pandemic beginning in late 2019. While children generally had lower rates of infection and morbidity compared with adults, there is data that pediatric oncology patients experience higher mortality compared with healthy children with rates ranging from 2.7 to $4.6 \%$ [40-43]. In addition to the acute mortality during infection, patients also experience substantial delays in their chemotherapy which may impact long-term survival [44]. There is currently no data on the efficacy of SARS-CoV-2 vaccination in pediatric oncology patients, but data from solid-organ transplant patients suggests that immunocompromised patients may benefit from a booster dose [45].

\section{Ancillary Measures}

In addition to antimicrobial prophylaxis and vaccination, there is substantial interest in other supportive care measures to reduce infection in pediatric oncology patients. This section will review the data on ethanol locks, CHB, GCSF, IVIG and mandatory hospitalization.

\section{Ethanol Locks}

Ethanol locks for central lines have been explored for primary and secondary prophylaxis in pediatric oncology patients with CVCs (Table 1). Ethanol locks involve infusing ethanol into a central line for 2 to $24 \mathrm{~h}$ with a goal of preventing and/or treating biofilm formation [46-48]. Primary prophylaxis with ethanol locks was explored in a prospective randomized-controlled trial in pediatric oncology patients ages 1-18 years of age. In this study, 307 patients were enrolled to receive either $70 \%$ ethanol or heparin locks. The patients who received ethanol locks showed a reduced rate of central line infections relative to those who received heparin and there were no apparent adverse events; however, the attrition rate from the study was high and therefore, there remains the potential for missed adverse events in the intervention group. This is particularly relevant as patients in the ethanol arm reported increased transient symptoms such as dizziness, taste alteration, blushing, and nausea [47].

Following salvage treatment of a central line infection, there is an inherent concern for relapse given the presence of colonization or biofilms, which are relatively resistant to systemic antibiotic treatment $[48,49]$. Wolf et al. conducted a randomized, controlled trial assessing the role of ethanol locks for secondary prophylaxis and treatment in 95 pediatric oncology patients with an identified central line infection compared with standard systemic antibiotic therapy. The trial was halted secondary to futility when the failure rate was equal within the ethanol lock and placebo groups (44\% vs $43 \%$, respectively). The authors concluded that ethanol locks were not effective for secondary prophylaxis or treatment among pediatric oncology patients with central-line associated bloodstream infections (CLABSI) relative to standard heparinized saline [48, 50]. In addition, children in the ethanol lock arm had an increased rate of central line occlusion compared to those who received heparin alone [48]. Schoot et al. did not report an increased incidence of central line occlusion in their trial of primary prophylaxis [47]; however, the use of ethanol locks should be carefully considered as central line occlusion is a highly problematic issue within pediatric oncology [51].

\section{Chlorhexidine Gluconate Baths}

Chlorhexidine gluconate ( $\mathrm{CHG})$ is an antiseptic which is bactericidal to gram-positive and gram-negative bacteremia. Chlorhexidine gluconate baths (CHB) involve cleansing the patient's body each day with CHG-impregnated wipes. Literature from pediatric patients with temporary CVCs inserted during critical care admissions suggests that $\mathrm{CHB}$ 
may have a role in reducing the rate of CLABSIs in these patients [52]. This has sparked interest within the pediatric oncology community given the heightened infectious risk and long-term use of CVCs within this patient population (Table 1). A retrospective analysis of 330 pediatric oncology patients reviewed infection rates in inpatients before and after implementation of a CHB regimen. While their results did not reach statistical significance across age groups, the rate of infection in patients ages 12-21 years of age showed a significant reduction in those who used CHB [53]. A recent COG trial, ACCL1034, investigated CHB using a randomized, double-blind design in children with external CVCs. The trial was closed early secondary to low accrual and posthoc analysis was conducted on the existing data. CHB did not reduce the rate of CLABSI in this patient population with 5.44 CLABSIs per 1000 central-line days in the CHB group compared with 3.10 in the control group $(\mathrm{p}=0.049)$ $[54,55]$. In addition, $24 \%$ patients who used CHB had a cutaneous adverse event [55]. In summary, there remains a paucity of data for the use of CHB in pediatric oncology patients and, therefore, consideration for use must weigh the risks and benefits accordingly.

\section{GCSF}

Risk of bacterial and fungal infections is associated with the duration and severity of neutropenia [15•, 20]. Thus, colony stimulating factors such as GCSF have been investigated in children with AML to minimize the duration of severe myelosuppression associated with these protocols (Table 1). In adult patients with AML, a meta-analysis demonstrated no impact on the rate of remission or infectious complications with routine use of GCSF following completion of induction chemotherapy [56]. There are conflicting reports in pediatrics. Sung et al. completed a survey of COG sites reviewing institutional practices for infection prophylaxis in children undergoing treatment for AML enrolled on AAML0531. They found that GCSF significantly reduced the number of bacterial infections and CDI in AML patients [57]. Previous to this, AML-BFM 98 prospectively randomized patients to understand the role of GCSF to augment hematologic recovery and decrease the risk of infectious complications. Importantly, while the duration of neutropenia was decreased in patients who received GCSF, the rate of febrile neutropenia and infection did not differ between groups; therefore, based on these results, routine GCSF use for primary prophylaxis was not recommended [58]. In addition, Ehlers et al. reported that in a subset of pediatric AML patients with over expression of G-CSFR Isoform IV, the use of prophylactic GCSF significantly increased their risk of leukemic relapse [59]. The adult meta-analysis does not support an associated increased relapse rate in patients in whom GCSF was used [56]. GCSF is a well-supported strategy for patients with hematologic malignancies and established infection to lessen the duration of neutropenia where the risk-benefit association is clearer [60], but there remains a lack of data to support its use for primary prophylaxis.

\section{IVIG}

IVIG is a pooled and concentrated blood product made of up human immunoglobulin, predominantly IgG. Hypogammaglobulinemia associated with hematologic malignancies is a focus of supportive care with primary or secondary prophylactic IVIG infusions. Supplementation with IVIG in pediatric patients has increased over time as infection risk secondary to hypogammaglobulinemia has been increasingly recognized [61, 62]. Prophylactic IVIG supplementation in pediatric oncology patients is highly variable, as initial evidence was derived from adult oncology literature in patients with B-cell chronic lymphocytic leukemia [63]. Van Tilburg et al. demonstrated that even during periods of decreased chemotherapy intensity, there remains a relative depression of specific antibodies which may denote increased susceptibility to infection [64]. Many clinical trials recommend enhanced supportive care for infant and Down syndrome patients with secondary hypogammaglobulinemia undergoing chemotherapy [62]. Supplementation in other populations is highly variable. In a recent retrospective review, Holmes et al. examined the frequency of febrile episodes in pediatric patients with ALL in the maintenance phase of their chemotherapy (Table 1). They report that while the frequency of fevers in the IgG-monitored population was higher than those not receiving supplementation, IVIG supplementation did not decrease the frequency of fevers, upper respiratory tract infections or bacteremia. Given the retrospective nature of the review, it is possible that monitoring was more aggressively undertaken in patients who were more susceptible to infection and toxicity at baseline [65]. In a review of 383 pediatric patients with ALL, Edington and colleagues found that rates of emergency department visits, hospitalization days, febrile neutropenia episodes, and severe infections were lower during IVIG supplemented days vs. non-supplemented days [66]. This work points to the potential positive impact of IVIG in patients receiving ALL therapy; however, further research is needed to delineate the larger role for primary prophylaxis in this population.

\section{Hospitalization}

Three studies have investigated whether continued inpatient hospitalization during periods of neutropenia improves outcomes in pediatric patients with AML (Table 1). In a secondary analysis of an upfront COG treatment trial (AAML0531), Sung et al. found that mandatory hospitalization did not reduce infection or non-relapse mortality, but 
did increase rates of CDI [57]. Miller et al. also found no difference in mortality in those who were discharged during periods of neutropenia. However, they did find that patients who were discharged early experienced increased rates of viridans group streptococcus, hypoxia, and hypotension on readmission [67]. In a prospective cohort of 573 patients with newly diagnosed AML, Getz et al. found that patients who were discharged from the hospital during periods of neutropenia did not have increased rates of bacteremia, delay in time to start of next course, or worse quality of life [68]. While inpatient hospitalization during neutropenia continues to be recommended for certain high-risk groups such as patients with AML and Down syndrome, there is limited data supporting this practice. Physicians and families should be aware that inpatient care has both potential benefits such as early response to subtle vital sign changes, but it also has potential harms such as increased rate of CDI and may not decrease the risk of infection.

\section{Special Populations}

Within pediatric oncology, there are several sub-populations that warrant particular attention regarding the infectious complications associated with their treatment. Among these populations are children with Down syndrome (DS), patients undergoing immunotherapy, adolescent and young adult patients, and patients with solid tumors.

\section{Immunotherapy}

The emergence of immunotherapy is changing the treatment paradigm for patients with both newly diagnosed and relapsed leukemia. There are no clinical trials evaluating infection prophylaxis in pediatric patients receiving blinatumomab, but multiple clinical trials have found that relapsed/refractory patients receiving blinatumomab are at lower risk for infectious toxicity compared to those receiving conventional chemotherapy $[69,70]$. Blinatumomab is also frequently used as bridging chemotherapy for patients recovering from a severe infection [71]. This data suggests that blinatumomab itself is not associated with increased risk of infection. However, it is important to consider the patient population and individual risk factors. For example, patients who have a history of multiple relapses, prior infections, or those with DS may still benefit from prophylaxis while receiving blinatumomab.

Emerging data suggests that pediatric patients receiving chimeric antigen receptor T-cell therapy (CAR-T) are at increased risk for infection, especially during the first month after infusion. In a single center retrospective review, $40 \%$ of patients experienced an infection within the 28 days after CAR-T cell infusion [72]. The majority of these were bacteremia (39\%) or respiratory viral infections (43\%). Prophylaxis regimens varied across patients. In a multicenter retrospective review, $40 \%$ of patients experienced an infection after CAR-T cell infusion [73]. The majority of these were bacterial or viral (50\% and $27 \%$ respectively) and $7 \%$ were fungal. Because the majority of patients currently receiving CAR-T cell therapy have relapsed or refractory disease, some centers may routinely use antibacterial prophylaxis based on the randomized trial by Alexander et al. which found the levofloxacin reduced bacteremia in children with relapsed ALL [15•]. Further studies into infection prophylaxis are needed in this high-risk patient population, especially as indications CAR-T cell therapy are expanding.

\section{Down syndrome}

Children with DS have a significantly increased risk of developing both AML and ALL relative to their peers [74]. In addition, when undergoing treatment with chemotherapy, they have been noted to have undue sensitivity to the agents used, leading to early identification of this group as high risk [75]. Heightened sensitivity to infections in children with DS extends beyond neutropenia and myelosuppression, as these children have alterations in humoral immune function, heightening their susceptibility to infectious complications while undergoing therapy $[76,77]$.

In ALL, this risk is twofold, as these children have a higher risk of relapse relative to non-DS patients as well as treatment-related mortality secondary to chemotherapy toxicity $[3,74,75,78]$. To characterize this in children with ALL, Rabin et al. described a small cohort of children with DS who had a disproportionate degree of neutropenia, mucositis, documented bacterial infections, and cellulitis relative to non-DS patients receiving ALL therapy [79]. Children with DS experience increased infectious complications during the induction and delayed intensification phases, but also during low intensity maintenance phases $[3,74,80]$. While presenting symptoms such as fever and hemodynamic instability were predictive of bacterial infection in patients with DS, children who presented within 7 days of receiving steroids also had an increased risk of bacterial infection [80]. In a retrospective review, Ceppi and colleagues noted that $27 \%$ of patients did not present with fever but had a subsequent diagnosis of bacterial infection [80]. While many adjustments have been made to the backbone of chemotherapy for children with DS and ALL, the heightened infectious risk is longitudinal throughout their treatment, which points to the importance of ongoing supportive care and heightened awareness of the potential complications of therapy. Recommended supportive care of children with DS has included IVIG supplementation, prophylactic antibiotic use, leucovorin 
rescue following intrathecal methotrexate, and hospital admission during high risk phases of treatment [75].

Children with DS and AML also experience a high rate of infectious complications while on chemotherapy. Evolution of clinical trials had led to the development of DSspecific protocols of lower intensity to mitigate the toxic effects of a standard backbone. Hassler et al. conducted a retrospective review describing 61 patients with DS who underwent treatment as per the reduced intensity AMLBFM-2004 protocol. Among these children, there were 157 infectious events including fever, as well as microbiologically documented or clinically documented infections. Nearly all bacterial isolates were gram positive, many of which were $S$. viridans. Viral infections were also significant among the described population. Therefore, despite the reduced intensity regimen, there remains significant infectious toxicity and mortality [81]. In contrast, a population study in Canada including children with DS did not report increased rates of infection or sepsis [82]. Further work by this group demonstrated that treatment on a DS-specific protocol was protective, with a significant reduction in sterile site infections and bacteremia. Steroid exposure was identified as a significant risk factor predisposing to infectious complications in concert with an AML backbone [83]. DS-specific protocols and supportive care guidelines may improve outcomes.

\section{Adolescents and Young Adults}

AYA oncology patients (ages 15-39) have inferior outcomes relative to both their primary oncologic disease as well as due to increased treatment-related mortality [84-86]. Larsen et al. reported that HR-ALL patients treated on the COG protocol AALL0232 had lower rates of febrile neutropenia $(35.6 \%$ vs $48.6 \% \mathrm{p}<0.0001)$ but an increased risk of death post-remission. Among these, 11 of 16 were secondary to infectious complications [84]. A data review from the Surveillance, Epidemiology, and End Results Program of AYA patients supports the finding of heightened noncancer related death in the AYA population. Importantly, infectious death was significantly elevated among all AYA patients with the most pronounced effect observed within patients with hematologic malignancy [86]. In an effort to understand the difference in infectious risk, Sano et al. compared antibiotic efficacy in the context of febrile neutropenia in children and AYA patients. They found that first-line antibiotic success rates (defined as resolution of fever and signs of infection) were lower in AYA patients compared to younger children $(53.8 \%$ vs. $63.7 \% \mathrm{p}=0.028)$ [85]. As with DS patients, AYA patients may benefit from specific protocol and supportive care modifications.

\section{Solid Tumors}

Patients with leukemia and lymphoma are at increased risk for infection compared to those with solid tumors $[9,10]$. It is, however, important to understand that children with solid tumors can also develop severe and life-threatening infections during therapy. Both surgery and radiation for local control result in disruption of the natural skin barrier with associated risk of infection. Implantation of medical devices such as prostheses and ventriculoperitoneal shunts are associated with both immediate and delayed infections $[87,88]$. And finally, tumors themselves can cause obstruction of critical structures and subsequent pneumonia, bowel perforation, urinary tract infection and cholangitis. Discussion regarding prevention of post-operative infections with peri-operative antibiotics and manipulation of prosthesis is outside the scope of this review, but there is data supporting this practice [87-90]. For patients with solid tumors, it is especially important to keep in mind the impact their tumor has on their underlying anatomy as well as recent surgeries and radiation.

\section{Conclusion}

Interpreting the data and implementing the clinical practice guidelines for infection prophylaxis in pediatric oncology can be challenging. To guide clinical decisions, it may help to keep several guiding principles in mind. First, patients with hematologic malignancies, those on high-dose corticosteroids, and those anticipated to have prolonged neutropenia are at highest risk for infection. Bacterial and fungal prophylaxis may be helpful in this population but warrants consideration of possible adverse effects, especially development of antimicrobial resistance. Second, the majority of ancillary measures do not have robust data to support routine use. However, they may be beneficial especially in high-risk groups such as DS and AYA patients. Third, more research is needed. This is especially critical given the increased utilization of immunotherapy in both relapsed and upfront treatment protocols. Continued rigorous clinical trials evaluating infection prophylaxis will allow us to continue to improve outcomes in childhood cancer.

\section{References}

Papers of particular interest, published recently, have been highlighted as:

- Of importance

$\bullet$ Of major importance 
1. Hunger SP, Lu X, Devidas M, et al. Improved survival for children and adolescents with acute lymphoblastic leukemia between 1990 and 2005: a report from the children's oncology group. J Clin Oncol. 2012;30(14):1663-9. https://doi.org/10.1200/JCO. 2011.37.8018.

2. Erdmann F, Frederiksen LE, Bonaventure A, et al. Childhood cancer: Survival, treatment modalities, late effects and improvements over time. Cancer Epidemiol. 2021;71(Pt B): 101733. https://doi.org/10.1016/j.canep.2020.101733.

3. O'Connor D, Bate J, Wade R, et al. Infection-related mortality in children with acute lymphoblastic leukemia: an analysis of infectious deaths on UKALL2003. Blood. 2014;124(7):1056-61. https://doi.org/10.1182/blood-2014-03-560847.

4. Calitri C, Ruberto E, Castagnola E. Antibiotic prophylaxis in neutropenic children with acute leukemia: Do the presently available data really support this practice? Eur J Haematol. 2018;101(6):721-7. https://doi.org/10.1111/ejh.13162.

5. King RN, Lager SL. Incidence of Clostridium difficile infections in patients receiving antimicrobial and acid-suppression therapy. Pharmacotherapy. 2011;31(7):642-8. https://doi.org/10. 1592/phco.31.7.642.

6. Bossù G, Di Sario R, Argentiero A, Esposito S. Antimicrobial Prophylaxis and Modifications of the Gut Microbiota in Children with Cancer. Antibiotics. 2021;10(2):152. https://doi.org/ 10.3390/antibiotics10020152.

7. Karol SE, Sun Y, Tang L, et al. Fluoroquinolone prophylaxis does not increase risk of neuropathy in children with acute lymphoblastic leukemia. Cancer Med. 2020;9(18):6550-5. https://doi.org/10.1002/cam4.3249.

8. Kelly M, Conway M, Wirth K, Potter-Bynoe G, Billett AL, Sandora TJ. Moving CLABSI prevention beyond the intensive care unit: risk factors in pediatric oncology patients. Infect Control Hosp Epidemiol. 2011;32(11):1079-85. https://doi.org/10.1086/ 662376.

9. Zajac-Spychala O, Wachowiak J, Gryniewicz-Kwiatkowska $\mathrm{O}$, et al. Prevalence, Epidemiology, Etiology, and Sensitivity of Invasive Bacterial Infections in Pediatric Patients Undergoing Oncological Treatment: A Multicenter Nationwide Study. Microb Drug Resist. 2021;27(1):53-63. https://doi.org/10.1089/ mdr.2019.0393.

10. Alexander S, Nieder M, Zerr DM, Fisher BT, Dvorak CC, Sung L. Prevention of bacterial infection in pediatric oncology: what do we know, what can we learn? Pediatr Blood Cancer. 2012;59(1):16-20. https://doi.org/10.1002/pbc.23416.

11. Gafter-Gvili A, Fraser A, Paul M, et al. Antibiotic prophylaxis for bacterial infections in afebrile neutropenic patients following chemotherapy. Cochrane Database Syst Rev. 2012;1:CD004386. https://doi.org/10.1002/14651858.CD004386.pub3.

12. Wolf J, Tang L, Flynn PM, et al. Levofloxacin Prophylaxis During Induction Therapy for Pediatric Acute Lymphoblastic Leukemia. Clin Infect Dis. 2017;65(11):1790-8. https://doi.org/10. 1093/cid/cix644

13. Widjajanto PH, Sumadiono S, Cloos J, Purwanto I, Sutaryo $\mathrm{S}$, Veerman AJ. Randomized double blind trial of ciprofloxacin prophylaxis during induction treatment in childhood acute lymphoblastic leukemia in the WK-ALL protocol in Indonesia. J Blood Med. 2013;4:1-9. https://doi.org/10.2147/JBM.S33906.

14. Laoprasopwattana K, Khwanna T, Suwankeeree P, Sujjanunt T, Tunyapanit W, Chelae S. Ciprofloxacin reduces occurrence of fever in children with acute leukemia who develop neutropenia during chemotherapy. Pediatr Infect Dis J. 2013;32(3):e94-8. https://doi.org/10.1097/INF.0b013e3182793610.

15.• Alexander S, Fisher BT, Gaur AH, et al. Effect of Levofloxacin Prophylaxis on Bacteremia in Children With Acute Leukemia or Undergoing Hematopoietic Stem Cell Transplantation: A Randomized Clinical Trial. JAMA. 2018;320(10):995-1004. https://doi.org/10.1001/jama.2018.12512. This randomized controlled trial of pediatric patients with AML or relapsed ALL found that levofloxacin prophylaxis was associated with decreased risk of bacteremia, decrease febrile neutropenia, and decreased exposure to broad spectrum antibiotics without increased risk of invasive fungal disease or $\mathrm{c}$. diff infection.

16. Egan G, Robinson PD, Martinez JPD, et al. Efficacy of antibiotic prophylaxis in patients with cancer and hematopoietic stem cell transplantation recipients: A systematic review of randomized trials. Cancer Med. 2019;8(10):4536-46. https://doi.org/10. 1002/cam4.2395.

17.••Lehrnbecher T, Fisher BT, Phillips B, et al. Guideline for Antibacterial Prophylaxis Administration in Pediatric Cancer and Hematopoietic Stem Cell Transplantation. Clin Infect Dis. 2020;71(1):226-36. https://doi.org/10.1093/cid/ciz1082. This systematic review provides clinical practice guidelines and a review of key clinical trial results for antibacterial prophylaxis in pediatric oncology patients.

18. Lehrnbecher T, Averbuch D, Castagnola E, et al. 8th European Conference on Infections in Leukaemia: 2020 guidelines for the use of antibiotics in paediatric patients with cancer or post-haematopoietic cell transplantation. Lancet Oncol. 2021;22(6):e270-80. https://doi.org/10.1016/S1470-2045(20) 30725-7.

19. Bartlett AW, Cann MP, Yeoh DK, et al. Epidemiology of invasive fungal infections in immunocompromised children; an Australian national 10-year review. Pediatr Blood Cancer. 2019;66(4):e27564. https://doi.org/10.1002/pbc.27564.

20. Fisher BT, Robinson PD, Lehrnbecher T, et al. Risk Factors for Invasive Fungal Disease in Pediatric Cancer and Hematopoietic Stem Cell Transplantation: A Systematic Review. J Pediatr Infect Dis Soc. 2018;7(3):191-8. https://doi.org/10.1093/jpids/pix030.

21. Donnelly JP, Chen SC, Kauffman CA, et al. Revision and Update of the Consensus Definitions of Invasive Fungal Disease From the European Organization for Research and Treatment of Cancer and the Mycoses Study Group Education and Research Consortium. Clin Infect Dis. 2020;71(6):1367-76. https://doi.org/10. 1093/cid/ciz1008

22. Mor M, Gilad G, Kornreich L, Fisher S, Yaniv I, Levy I. Invasive fungal infections in pediatric oncology. Pediatr Blood Cancer. 2011;56(7):1092-7. https://doi.org/10.1002/pbc.23005.

23. Fisher BT, Kavcic M, Li Y, et al. Antifungal prophylaxis associated with decreased induction mortality rates and resources utilized in children with new-onset acute myeloid leukemia. Clin Infect Dis. 2014;58(4):502-8. https://doi.org/10.1093/cid/cit781.

24. • Fisher BT, Zaoutis T, Dvorak CC, et al. Effect of Caspofungin vs Fluconazole Prophylaxis on Invasive Fungal Disease Among Children and Young Adults With Acute Myeloid Leukemia: A Randomized Clinical Trial. JAMA. 2019;322(17):1673-81. https://doi.org/10.1001/jama.2019.15702. This randomized controlled trial of pediatric patients with AML found that caspofungin prophylaxis was associated with a decreased risk of invasive fungal disease compared with fluconazole prophylaxis.

25.• Lehrnbecher T, Fisher BT, Phillips B, et al. Clinical Practice Guideline for Systemic Antifungal Prophylaxis in Pediatric Patients With Cancer and Hematopoietic Stem-Cell Transplantation Recipients. J Clin Oncol. 2020;38(27):3205-16. https:// doi.org/10.1200/JCO.20.00158. This systematic review provides clinical practice guidelines and a review of key clinical trial results for antifungal prophylaxis in pediatric oncology patients.

26. Groll AH, Pana D, Lanternier F, et al. 8th European Conference on Infections in Leukaemia: 2020 guidelines for the diagnosis, prevention, and treatment of invasive fungal diseases in 
paediatric patients with cancer or post-haematopoietic cell transplantation. Lancet Oncol. 2021;22(6):e254-69. https://doi.org/ 10.1016/S1470-2045(20)30723-3.

27. Hughes WT, Kuhn S, Chaudhary S, et al. Successful chemoprophylaxis for Pneumocystis carinii pneumonitis. N Engl J Med. 1977;297(26):1419-26. https://doi.org/10.1056/NEJM197712 292972602

28. Geerlinks AV, Campigotto A, Science M, Gupta S. Impact of prophylaxis choice on risk of pneumocystis pneumonia in children with cancer: A case-control study. Eur J Cancer. 2020;140:71-5. https://doi.org/10.1016/j.ejca.2020.09.013.

29. Prasad P, Nania JJ, Shankar SM. Pneumocystis pneumonia in children receiving chemotherapy. Pediatr Blood Cancer. 2008;50(4):896-8. https://doi.org/10.1002/pbc.21202.

30. Proudfoot R, Phillips B, Wilne S. Guidelines for the Prophylaxis of Pneumocystis jirovecii Pneumonia (PJP) in Children With Solid Tumors. J Pediatr Hematol Oncol. 2017;39(3):194-202. https://doi.org/10.1097/MPH.0000000000000771.

31. Werbel WA, Ison MG, Angarone MP, Yang A, Stosor V. Lymphopenia is associated with late onset Pneumocystis jirovecii pneumonia in solid organ transplantation. Transpl Infect Dis. 2018;20(3):e12876. https://doi.org/10.1111/tid.12876.

32. Dixon SB, Lane A, O'Brien MM, et al. Viral surveillance using PCR during treatment of AML and ALL. Pediatr Blood Cancer. 2018;65(1):e26752. https://doi.org/10.1002/pbc.26752.

33. Buus-Gehrig C, Bochennek K, Hennies MT, Klingebiel T, Groll $\mathrm{AH}$, Lehrnbecher T. Systemic viral infection in children receiving chemotherapy for acute leukemia. Pediatr Blood Cancer. 2020;67(12):e28673. https://doi.org/10.1002/pbc.28673.

34. Beverley M LP, Kaplan J, Turner N. Flu fighters: Flu vaccine administrations in pediatric hematology oncology patients. $\mathbf{J}$ Clin Oncol 2018;36(30). https://ascopubs.org/doi/abs/10.1200/ JCO.2018.36.30_suppl.131.

35. Goossen GM, Kremer LC, van de Wetering MD. Influenza vaccination in children being treated with chemotherapy for cancer. Cochrane Database Syst Rev. 2013;(8):CD006484. https://doi. org/10.1002/14651858.CD006484.pub3.

36. Reilly A, Kersun LS, McDonald K, Weinberg A, Jawad AF, Sullivan KE. The efficacy of influenza vaccination in a pediatric oncology population. J Pediatr Hematol Oncol. 2010;32(5):e177-81. https://doi.org/10.1097/MPH.0b013e3181 d869f3.

37. Hakim H, Allison KJ, Van de Velde LA, et al. Immunogenicity and safety of high-dose trivalent inactivated influenza vaccine compared to standard-dose vaccine in children and young adults with cancer or HIV infection. Vaccine. 2016;34(27):3141-8. https://doi.org/10.1016/j.vaccine.2016.04.053.

38. Kotecha RS, Wadia UD, Jacoby P, et al. Immunogenicity and clinical effectiveness of the trivalent inactivated influenza vaccine in immunocompromised children undergoing treatment for cancer. Cancer Med. 2016;5(2):285-93. https://doi.org/10.1002/ cam4.596.

39. Sykes A, Gerhardt E, Tang L, Adderson EE. The Effectiveness of Trivalent Inactivated Influenza Vaccine in Children with Acute Leukemia. J Pediatr. 2017;191:218-224 e1. https://doi.org/10. 1016/j.jpeds.2017.08.071

40. Madhusoodhan PP, Pierro J, Musante J, et al. Characterization of COVID-19 disease in pediatric oncology patients: The New York-New Jersey regional experience. Pediatr Blood Cancer. 2021;68(3):e28843. https://doi.org/10.1002/pbc.28843.

41. Vijenthira A, Gong IY, Fox TA, et al. Outcomes of patients with hematologic malignancies and COVID-19: a systematic review and meta-analysis of 3377 patients. Blood. 2020;136(25):288192. https://doi.org/10.1182/blood.2020008824.
42. UAB. The Pediatric COVID-19 Cancer Case Report. https:// www.uab.edu/medicine/icos/icos-research/the-pocc-report. Accessed Aug 2021.

43. Global SJ. Global Registry of COVID-19 in Pediatric Cancer. https://global.stjude.org/en-us/global-covid-19-observatoryandresource-center-for-childhood-cancer/registry.html. Accessed Aug 2021.

44. Johnston EE, Martinez I, Davis ES, Caudill C, Richman J, Brackett J, et al. SARS-CoV-2 in childhood cancer in 2020: a disease of disparities. J Clin Oncol. 2021;39(34):3778-88. https://doi. org/10.1200/JCO.21.00702.

45. Kamar N, Abravanel F, Marion O, Couat C, Izopet J, Del Bello A. Three Doses of an mRNA Covid-19 Vaccine in Solid-Organ Transplant Recipients. N Engl J Med. 2021;385(7):661-2. https://doi.org/10.1056/NEJMc2108861.

46. Wolf J, Allison KJ, Tang L, Sun Y, Hayden RT, Flynn PM. No evidence of benefit from antibiotic lock therapy in pediatric oncology patients with central line-related bloodstream infection: results of a retrospective matched cohort study and review of the literature. Pediatr Blood Cancer. 2014;61(10):1811-5. https://doi.org/10.1002/pbc.25101.

47. Schoot RA, van Ommen CH, Stijnen T, et al. Prevention of central venous catheter-associated bloodstream infections in paediatric oncology patients using 70\% ethanol locks: A randomised controlled multi-centre trial. Eur J Cancer. 2015;51(14):2031-8. https://doi.org/10.1016/j.ejca.2015.06.126.

48. Wolf J, Connell TG, Allison KJ, et al. Treatment and secondary prophylaxis with ethanol lock therapy for central line-associated bloodstream infection in paediatric cancer: a randomised, double-blind, controlled trial. Lancet Infect Dis. 2018;18(8):854-63. https://doi.org/10.1016/S1473-3099(18)30224-X.

49. Lebeaux D, Ghigo JM, Beloin C. Biofilm-related infections: bridging the gap between clinical management and fundamental aspects of recalcitrance toward antibiotics. Microbiol Mol Biol Rev. 2014;78(3):510-43. https://doi.org/10.1128/MMBR. 00013-14.

50. Wolf J, Connell TG, Curtis N, Flynn PM. Ethanol lock therapy. Lancet Infect Dis. 2018;18(12):1306. https://doi.org/10.1016/ S1473-3099(18)30678-9.

51. Rickard CM, Ullman AJ. Bloodstream infection and occlusion of central venous catheters in children. Lancet Infect Dis. 2018;18(8):815-7. https://doi.org/10.1016/S1473-3099(18) 30267-6.

52. Kim HY, Lee WK, Na S, Roh YH, Shin CS, Kim J. The effects of chlorhexidine gluconate bathing on health care-associated infection in intensive care units: A meta-analysis. J Crit Care. 2016;32:126-37. https://doi.org/10.1016/j.jcrc.2015.11.011.

53. Raulji CM, Clay K, Velasco C, Yu LC. Daily Bathing with Chlorhexidine and Its Effects on Nosocomial Infection Rates in Pediatric Oncology Patients. Pediatr Hematol Oncol. 2015;32(5):315-21. https://doi.org/10.3109/08880018.2015. 1013588 .

54. Zerr DM, Milstone AM, Adler AL, Dvorak CC, Fisher BT, Sung L. Reply to Are we certain that chlorhexidine gluconate bathing is not beneficial in deducing central line associated blood stream infections among children with cancer or undergoing hematopoietic stem cell transplantation? Cancer. 2021;127(15):2813-4. https://doi.org/10.1002/cncr.33570.

55. Zerr DM, Milstone AM, Dvorak CC, et al. Chlorhexidine gluconate bathing in children with cancer or those undergoing hematopoietic stem cell transplantation: A double-blinded randomized controlled trial from the Children's Oncology Group. Cancer. 2020;127(1):56-66. https://doi.org/10.1002/cncr.33271.

56. Gurion R, Belnik-Plitman Y, Gafter-Gvili A, et al. Colonystimulating factors for prevention and treatment of infectious 
complications in patients with acute myelogenous leukemia. Cochrane Database Syst Rev. 2012;6:CD008238. https://doi. org/10.1002/14651858.CD008238.pub3.

57. Sung L, Aplenc R, Alonzo TA, Gerbing RB, Lehrnbecher T, Gamis AS. Effectiveness of supportive care measures to reduce infections in pediatric AML: a report from the Children's Oncology Group. Blood. 2013;121(18):3573-7. https:// doi.org/10.1182/blood-2013-01-476614.

58. Lehrnbecher T, Zimmermann M, Reinhardt D, Dworzak M, Stary J, Creutzig U. Prophylactic human granulocyte colonystimulating factor after induction therapy in pediatric acute myeloid leukemia. Blood. 2007;109(3):936-43. https://doi. org/10.1182/blood-2006-07-035915.

59. Ehlers S, Herbst C, Zimmermann M, et al. Granulocyte colony-stimulating factor (G-CSF) treatment of childhood acute myeloid leukemias that overexpress the differentiation-defective G-CSF receptor isoform IV is associated with a higher incidence of relapse. J Clin Oncol. 2010;28(15):2591-7. https://doi.org/10.1200/JCO.2009.25.9010.

60. Grigull L, Beilken A, Schmid H, et al. Secondary prophylaxis of invasive fungal infections with combination antifungal therapy and G-CSF-mobilized granulocyte transfusions in three children with hematological malignancies. Support Care Cancer. 2006;14(7):783-6. https://doi.org/10.1007/s00520-005-0910-8.

61. Balch A, Wilkes J, Thorell E, Pavia A, Sherwin CMT, Enioutina EY. Changing trends in IVIG use in pediatric patients: A retrospective review of practices in a network of major USA pediatric hospitals. Int Immunopharmacol. 2019;76:105868. https://doi. org/10.1016/j.intimp.2019.105868.

62. Edington HJ, Sutton KS, Bennett C, Chandrakasan S, SternerAllison J, Castellino SM. Dealing with a critical national shortage-Approaches to triaging immune globulin supply in pediatric hematology and oncology. Pediatr Blood Cancer. 2020;67(7):e28260. https://doi.org/10.1002/pbc.28260.

63. Raanani P, Gafter-Gvili A, Paul M, Ben-Bassat I, Leibovici L, Shpilberg O. Immunoglobulin prophylaxis in chronic lymphocytic leukemia and multiple myeloma: systematic review and meta-analysis. Leuk Lymphoma. 2009;50(5):764-72. https://doi. org/10.1080/10428190902856824.

64. van Tilburg CM, Bierings MB, Berbers GA, et al. Impact of treatment reduction for childhood acute lymphoblastic leukemia on serum immunoglobulins and antibodies against vaccinepreventable diseases. Pediatr Blood Cancer. 2012;58(5):701-7. https://doi.org/10.1002/pbc.23258.

65. Holmes EA, Friedman DL, Connelly JA, Dulek DE, Zhao Z, Esbenshade AJ. Impact of IgG Monitoring and IVIG Supplementation on the Frequency of Febrile Illnesses in Pediatric Acute Lymphoblastic Leukemia Patients Undergoing Maintenance Chemotherapy. J Pediatr Hematol Oncol. 2019;41(6):4238. https://doi.org/10.1097/MPH.0000000000001415.

66. Edington HJDN, Miller T, et al. Predictors and Outcomes of Immunoglobulin Supplementation in B-cell Acute Lymphoblastic Leukemia. Pediatr Blood Cancer. 2021;68:e2960.

67. Miller TP, Getz KD, Kavcic M, et al. A comparison of discharge strategies after chemotherapy completion in pediatric patients with acute myeloid leukemia: a report from the Children's Oncology Group. Leuk Lymphoma. 2016;57(7):1567-74. https://doi.org/10.3109/10428194.2015.1088652.

68. Getz KD LY, Szymczak JE, Aftandilian C, Arnold SD, O Bona K, Caywood , E CA, Gramatges MM, Henry M, Lotterman C, Maloney K, Mian A, Mody R, Raetz EA, Rubnitz J, Verma A, Winick N, Wilkes JJ, Yu JC, Fisher BT, Aplenc R. Home or Away from Home: A Multi-Institution Study Comparing Medical Outcomes, Patient Perspectives, and Health-Related Quality of Life for Outpatient Versus Inpatient Management after
Chemotherapy for Pediatric Acute Myeloid Leukemia. Blood. 2019;2019(134)(1). https://doi.org/10.1182/blood-2019-131761.

69. Brown PA, Ji L, Xu X, et al. Effect of Postreinduction Therapy Consolidation With Blinatumomab vs Chemotherapy on Disease-Free Survival in Children, Adolescents, and Young Adults With First Relapse of B-Cell Acute Lymphoblastic Leukemia: A Randomized Clinical Trial. JAMA. 2021;325(9):833-42. https:// doi.org/10.1001/jama.2021.0669.

70. Locatelli F, Zugmaier G, Rizzari C, et al. Effect of Blinatumomab vs Chemotherapy on Event-Free Survival Among Children With High-risk First-Relapse B-Cell Acute Lymphoblastic Leukemia: A Randomized Clinical Trial. JAMA. 2021;325(9):843-54. https://doi.org/10.1001/jama.2021.0987.

71. Contreras CF, Higham CS, Behnert A, Kim K, Stieglitz E, Tasian SK. Clinical utilization of blinatumomab and inotuzumab immunotherapy in children with relapsed or refractory B-acute lymphoblastic leukemia. Pediatr Blood Cancer. 2021;68(1):e28718. https://doi.org/10.1002/pbc.28718.

72. Vora SB, Waghmare A, Englund JA, Qu P, Gardner RA, Hill JA. Infectious Complications Following CD19 Chimeric Antigen Receptor T-cell Therapy for Children, Adolescents, and Young Adults. Open Forum Infect Dis. 2020;7(5):ofaa121. https://doi. org/10.1093/ofid/ofaa121.

73. Moskop AVM, Prabhu S, Phillips CL, Stefanski HE, Talano JA, Baggott C, Pacenta HL, Rossoff J, Margossian S, Myers D, Brown PA, Hermiston M, Krukski MC, Wilcox R, Fabrizio VA, Goksenin Y, Karras N, Qayed M, Satwani P, Keating AK, Rabik CA, Chinnabhandar V, Curran KJ, Mackall C, Laetsch TW, Schultz L. Immune Reconstitution and Infections in the Real-World Use of Tisagenlecleucel in Pediatric and Young Adult ALL. Transplant Cell Ther Meet. 2021. https://ash.confex. com/ash/2021/webprogram/Paper146393.html.

74. Buitenkamp TD, Izraeli S, Zimmermann M, et al. Acute lymphoblastic leukemia in children with Down syndrome: a retrospective analysis from the Ponte di Legno study group. Blood. 2014;123(1):70-7. https://doi.org/10.1182/ blood-2013-06-509463.

75. Salazar EG, Li Y, Fisher BT, et al. Supportive care utilization and treatment toxicity in children with Down syndrome and acute lymphoid leukaemia at free-standing paediatric hospitals in the United States. Br J Haematol. 2016;174(4):591-9. https:// doi.org/10.1111/bjh.14085.

76. Bloemers BL, van Bleek GM, Kimpen JL, Bont L. Distinct abnormalities in the innate immune system of children with Down syndrome. J Pediatr. 2010;156(5):804-9, 809 e1-809 e5. https://doi.org/10.1016/j.jpeds.2009.12.006.

77. Kusters MA, Verstegen RH, Gemen EF, de Vries E. Intrinsic defect of the immune system in children with Down syndrome: a review. Clin Exp Immunol. 2009;156(2):189-93. https://doi. org/10.1111/j.1365-2249.2009.03890.x.

78. Goodman EKLY, Seif AE, et al. Treatment toxicity and supportive care utilization in children with Down Syndrome and acute lymphoid leukemia at free-standing pediatric hospitals in the United States. Blood. 2014;124(21):553.

79. Rabin KR, Smith J, Kozinetz CA. Myelosuppression and infectious complications in children with Down syndrome and acute lymphoblastic leukemia. Pediatr Blood Cancer. 2012;58(4):6335. https://doi.org/10.1002/pbc.23371.

80. Ceppi F, Stephens D, den Hollander BS, et al. Clinical presentation and risk factors of serious infections in children with Down syndrome treated for acute lymphoblastic leukemia. Pediatr Blood Cancer. 2016;63(11):1949-53. https://doi.org/10.1002/ pbc.26127.

81. Hassler A, Bochennek K, Gilfert J, et al. Infectious Complications in Children With Acute Myeloid Leukemia and Down Syndrome: Analysis of the Prospective Multicenter Trial AML-BFM 
2004. Pediatr Blood Cancer. 2016;63(6):1070-4. https://doi.org/ 10.1002/pbc.25917.

82. Dix D, Cellot S, Price V, et al. Association between corticosteroids and infection, sepsis, and infectious death in pediatric acute myeloid leukemia (AML): results from the Canadian infections in AML research group. Clin Infect Dis. 2012;55(12):1608-14. https://doi.org/10.1093/cid/cis774.

83. Tran TH, Mitchell D, Dix D, et al. Infections in children with down syndrome and acute myeloid leukemia: a report from the Canadian infections in AML research group. Infect Agent Cancer. 2013;8(1):47. https://doi.org/10.1186/1750-9378-8-47.

84. Larsen ECSW, Nachman J, et al. Treatment toxicity in adolescents and young adults (AYA) patients compared with younger patients treatment for high risk B-precursor acute lymphoblastic leukemia: A report from the Children's Oncology Group Study AALL0232. Blood. 2011;118(21):1510.

85. Sano H, Kobayashi R, Suzuki D, et al. Differential efficacy of empirical antibiotic therapy for febrile neutropenia in adolescent/young adult (AYA) and child patients. Int J Hematol. 2018;108(5):543-9. https://doi.org/10.1007/s12185-018-2503-6.

86. Anderson C, Lund JL, Weaver MA, Wood WA, Olshan AF, Nichols HB. Noncancer mortality among adolescents and young adults with cancer. Cancer. 2019;125(12):2107-14. https://doi. org/10.1002/cncr.32063.

87. Harrison DJ, Geller DS, Gill JD, Lewis VO, Gorlick R. Current and future therapeutic approaches for osteosarcoma. Expert Rev Anticancer Ther. 2018;18(1):39-50. https://doi.org/10.1080/ 14737140.2018.1413939.

88. Raygor KP, Oh T, Hwang JY, et al. Ventriculoperitoneal shunt infection rates using a standard surgical technique, including topical and intraventricular vancomycin: the Children's Hospital Oakland experience. J Neurosurg Pediatr. 2020:1-9. https://doi. org/10.3171/2020.4.PEDS209.

89. Smolle MA, Andreou D, Tunn PU, Leithner A. Advances in tumour endoprostheses: a systematic review. EFORT Open Rev. 2019;4(7):445-59. https://doi.org/10.1302/2058-5241.4.180081.

90. Khoshbin A, So JP, Aleem IS, et al. Antibiotic Prophylaxis to Prevent Surgical Site Infections in Children: A Prospective Cohort Study. Ann Surg. 2015;262(2):397-402. https://doi.org/ 10.1097/SLA.0000000000000938.

Publisher's Note Springer Nature remains neutral with regard to jurisdictional claims in published maps and institutional affiliations. 